Stud. Univ. Babeş-Bolyai Math. 66(2021), No. 1, 105-113

DOI: 10.24193/subbmath.2021.1.09

\title{
Fredholm and Volterra nonlinear possibilistic integral equations
}

\author{
Sorin G. Gal and Ionuţ T. Iancu
}

Dedicated to Professor Gheorghe Moroşanu on the occasion of his 70th anniversary.

\begin{abstract}
In this paper we study the nonlinear functional equations obtained from the classical integral equations of Fredholm and of Volterra of second kind, by replacing there the linear Lebesgue integral with the nonlinear possibilistic integral.
\end{abstract}

Mathematics Subject Classification (2010): 45B05, 47H10, 28E10, 28A99.

Keywords: Possibility measure, nonlinear possibilistic integral, Fredholm nonlinear possibilistic integral equation, Volterra nonlinear possibilistic integral equation, fixed point theorem, successive approximations.

\section{Introduction}

It is known that possibility theory is an alternative theory to the probability theory, dealing with certain types of uncertainty and treatment of incomplete information (see, e.g., [2], [4]). In the possibilistic models, all the probabilistic indicators (like expected value, variance, probability measure, integral with respect to a measure, etc) are replaced with suitable possibility indicators. These analogies allow to extend many classical results based on probability theory, to the possibilistic frame. We can mention here the contributions of the first named author to results concerning approximation by possibilistic operators (called also max-product operators), see [1], [3], [7], [9] or to the possibilistic laws of large numbers, see, e.g., [8], and the references therein.

In this paper we continue our researches in this directions, by extending in the frame of possibility theory, results concerning classical integral equations.

In this sense, it is natural and of interest to replace in the classical integral equations, the linear Lebesgue integral by various other kinds of nonlinear integrals. Thus, in the very recent papers [5], [6], the first named author has replaced the linear Lebesgue integral by its non linear extension called Choquet integral and studied 
the existence of the solutions for the corresponding Fredholm-Choquet and VolterraChoquet integral equations.

In this spirit of ideas, we study here the nonlinear equations obtained by replacing in the classical Fredholm and Volterra integral equations, the linear Lebesgue integral with the nonlinear possibilistic integral $(P o s) \int$ with respect to a possibility measure $P_{\lambda}$ generated by the possibility distribution $\lambda$. More exactly, we study the nonlinear possibilistic integral equations

$$
\varphi(x)=f(x)+\alpha \cdot(\text { Pos }) \int_{\Omega} K(x, s) \varphi(s) d P_{\lambda}(s), x \in \Omega,
$$

with the given data $\alpha \in \mathbb{R}, f: \Omega \rightarrow \mathbb{R}, K: \Omega \times \Omega \rightarrow \mathbb{R}$ and the unknown function $\varphi: \Omega \rightarrow \mathbb{R}$ in the case of Fredholm type equation, and by

$$
\varphi(x)=f(x)+\alpha \cdot(P o s) \int_{a}^{x} K(x, s, \varphi(s)) d P_{\lambda}(s), x \in[a, b],
$$

with the given data $\alpha \in \mathbb{R}, f:[a, b] \rightarrow \mathbb{R}, K:[a, b] \times[a, b] \times \mathbb{R} \rightarrow \mathbb{R}$ and the unknown function $\varphi:[a, b] \rightarrow \mathbb{R}$, in the case of Volterra type equation.

As we will see, due to the definition of the possibilistic integral, in fact we obtain functional equations which have solutions under some additional conditions. Also, it is worth mentioning that while the classical Fredholm and Volterra integral equations are linear, due to the nonlinearity of the possibilistic integral, obviously that the Fredholm and Volterra possibilistic integral equations are nonlinear.

Section 2 contains some preliminaries on the possibility measures and integrals we will need in the next sections. In Section 3, the existence and construction of the solutions for the Fredholm nonlinear possibilistic integral equation (1.1). Thus, for $P_{\lambda}$ belonging to large classes of possibility measures, we show that this functional equation has solutions under some appropriate conditions (similar to those in the classical case) on the given data $f, \alpha$ and $K$.

Finally, in Section 4 we study the existence of the solutions for the Volterra nonlinear possibilistic integral type equation (1.2).

\section{Preliminaries on possibility measures and integrals}

Firstly, we summarize some known concepts in possibility theory, which will be used in the next sections. For details, see e.g. [4] or [2].

Definition 2.1. Let $\Omega$ be a non-empty set.

(i) A possibilistic (fuzzy) variable $X$ is simply an application $X: \Omega \rightarrow \mathbb{R}$.

(ii) A possibility distribution (on $\Omega$ ), is a function $\lambda: \Omega \rightarrow[0,+\infty)$, such that $\sup \{\lambda(s) ; s \in \Omega\}=V<+\infty$. If $V=1$, then $\lambda$ it is called normalized possibility distribution.

(iii) A possibility measure is a mapping $P: \mathcal{P}(\Omega) \rightarrow[0,+\infty)$, satisfying the axioms $P(\emptyset)=0, P(\Omega)=1$ and $P\left(\bigcup_{i \in I} A_{i}\right)=\sup \left\{P\left(A_{i}\right) ; i \in I\right\}$ for all $A_{i} \in \Omega$, and any $I$, family of indices. 
It is well-known (see e.g. [4]) that any possibility distribution $\lambda$ on $\Omega$, induces a possibility measure $P_{\lambda}$, given by the formulas $P_{\lambda}(A)=\sup \{\lambda(s) ; s \in A\}$, for all $A \subset \Omega, A \neq \emptyset, P_{\lambda}(\emptyset)=0$.

(iv) (see e.g. [2]) The possibilistic integral of $f: \Omega \rightarrow \mathbb{R}_{+}$on $A \subset \Omega$, with respect to the possibilistic measure $P_{\lambda}$ induced by the possibilistic distribution $\lambda$, is defined by

$$
(\text { Pos }) \int_{A} f(t) d P_{\lambda}(t)=\sup \{f(t) \cdot \lambda(t) ; t \in A\} .
$$

It is clear that this definition is a particular case of the $t$-possibilistic integral with respect to a semi-norm $t$, introduced in [2], by taking there $t(x, y)=x \cdot y$.

(v) The following properties hold : for all $f, g \geq 0$ and $c \in \mathbb{R}, c \geq 0$

$$
\begin{gathered}
(\text { Pos }) \int_{A}(f(s)+g(s)) d P_{\lambda}(s) \leq(\text { Pos }) \int_{A} f(s) d P_{\lambda}(s)+(\text { Pos }) \int_{A} g(s) d P_{\lambda}(s), \\
(\text { Pos }) \int_{A}[c f(s)] d P_{\lambda}(s)=c \cdot(\text { Pos }) \int_{A} f(s) d P_{\lambda}(s) .
\end{gathered}
$$

\section{Fredholm possibilistic integral equations}

Let us denote by $B_{+}(\Omega)$, the Banach space of all positive and bounded functions $f: \Omega \rightarrow \mathbb{R}_{+}$, endowed with the uniform norm, denoted here by $\|\cdot\|$. It is clear that $B_{+}(\Omega)$ endowed with the metric generated by the uniform norm, is a complete metric space.

Taking into account the definition of the possibilistic integral in Definition 2.1, the Fredholm possibilistic integral equation in (1.1), formally becomes the nonlinear functional equation

$$
\varphi(x)=f(x)+\alpha \cdot \sup \{K(x, s) \cdot \varphi(s) \cdot \lambda(s) ; s \in \Omega\}, x \in \Omega .
$$

The first main result is the following.

Theorem 3.1. Let $\Omega \neq \emptyset$ and $P_{\lambda}$ be possibility measure induced by the possibility distribution $\lambda$ on $\Omega$.

Let us also suppose that

$$
0 \leq K(x, s) \leq M<+\infty, \text { for all } x, s \in \Omega .
$$

Then, for any $f \in B_{+}(\Omega)$ and any $0<\alpha<\frac{1}{M}$, the Fredholm possibilistic functional equation (3.1) has a unique solution $\varphi^{*} \in B_{+}(\Omega)$.

Moreover, denoting

$$
A(\varphi)(x)=f(x)+\alpha \cdot \sup \{K(x, s) \lambda(s) \cdot \varphi(s) ; s \in \Omega\} \text { and } A^{n}\left(\varphi_{0}\right)=A\left[A^{n-1}\left(\varphi_{0}\right)\right],
$$

for any arbitrary $\varphi_{0} \in B_{+}(\Omega)$, the following estimate holds

$$
\left\|A^{n}\left(\varphi_{0}\right)-\varphi^{*}\right\| \leq \frac{\alpha \cdot M}{1-\alpha \cdot M} \cdot\left\|A\left(\varphi_{0}\right)-\varphi_{0}\right\| .
$$


Proof. For any $x \in \Omega$ fixed and $\varphi \in B_{+}(\Omega)$, let us denote

$$
T(\varphi)(x)=\sup \{K(x, s) \varphi(s) \lambda(s) ; s \in \Omega\} .
$$

By hypothesis we immediately get $T(\varphi) \in B_{+}(\Omega)$.

This implies that $A(\varphi)(x)=f(x)+\alpha \cdot T(\varphi)(x) \in B_{+}(\Omega)$, for all $0 \leq \alpha<\infty$.

Let $\varphi, \psi \in B_{+}(\Omega)$. We have $\varphi=\varphi-\psi+\psi \leq|\varphi-\psi|+\psi$, which successively implies

$$
T(\varphi)(x) \leq T(|\varphi-\psi|)(x)+T(\psi)(x)
$$

that is

$$
T(\varphi)(x)-T(\psi)(x) \leq T(|\varphi-\psi|)(x) .
$$
it follows

Writing now $\psi=\psi-\varphi+\varphi \leq|\varphi-\psi|+\varphi$ and applying the above reasonings,

$$
T(\psi)(x)-T(\varphi)(x) \leq T(|\varphi-\psi|)(x),
$$

which combined with the above inequality gives

$$
|T(\varphi)(x)-T(\psi)(x)| \leq T(|\varphi-\psi|)(x),
$$

that is

$$
\begin{aligned}
& \mid \sup \{K(x, s) \psi(s) \lambda(s) ; s \in \Omega\}-\sup \{K(x, s) \varphi(s) \lambda(s) ; s \in \Omega\} \mid \\
& \leq \sup \{|K(x, s) \psi(s) \lambda(s)-K(x, s) \varphi(s) \lambda(s)| ; s \in \Omega\} .
\end{aligned}
$$

Since for all $x \in \Omega$ we have

$$
\begin{aligned}
|A(\varphi)(x)-A(\psi)(x)| & =\alpha \cdot|T(\varphi)(x)-T(\psi)(x)| \leq \alpha \cdot T(|\varphi-\psi|)(x) \\
& \leq M \cdot \alpha \cdot\|\varphi-\psi\|,
\end{aligned}
$$

passing to supremum after $x \in \Omega$, we immediately obtain

$$
d(A(\varphi), A(\psi)):=\|A(\varphi)-A(\psi)\| \leq M \cdot \alpha \cdot\|\varphi-\psi\|:=M \cdot \alpha \cdot d(\varphi, \psi) .
$$

The hypothesis implies that $d: B_{+}(\Omega) \times B_{+}(\Omega) \rightarrow \mathbb{R}_{+}$is a contraction on the complete metric space $B_{+}(\Omega)$ endowed with the metric $d(\varphi, \psi)=\|\varphi-\psi\|$, which by the Banach's fixed point theorem implies the desired conclusion.

Remark 3.2. In general, under the conditions in Theorem 3.1 the sequence of successive approximation cannot be written in the explicit form as in the classical linear Fredholm integral equation (i.e. by using the so called resolvent). However, under some additional hypothesis on the input data $f, \lambda$ and $K$, this can be done, exemplified by the following result.

Corollary 3.3. Let $\Omega=[a, b]$ and $P_{\lambda}$ be the possibility measure induced by the possibility distribution $\lambda$, supposed to be nondecreasing on $\Omega$.

Let us suppose that $\alpha>0, f(x) \geq 0, K(x, s) \geq 0$, for all $x, s \in[a, b], K(b, b)>0$,

$$
K(\cdot, \cdot) \text { is nondecreasing in each variable on }[a, b] \text {, }
$$

$$
f \text { is nondecreasing on }[a, b] \text {. }
$$

Then, for any $\alpha<\frac{1}{K(b, b)}$, the Fredholm possibilistic functional equation (3.1) has a unique solution $\varphi^{*} \in B_{+}[a, b]$, nondecreasing on $[a, b]$. 
Moreover, denoting $K_{1}(x, t)=K(x, t)$ and by the recurrence formula

$$
\begin{gathered}
K_{j}(x, t)=(\text { Pos }) \int_{a}^{b} K_{j-1}(x, s) K(s, t) d P_{\lambda}(s) \\
=\sup \left\{K_{j-1}(x, s) K(s, t) \cdot \lambda(s) ; s \in[a, b]\right\}, j \in \mathbb{N}, j \geq 2,
\end{gathered}
$$

for the sequence of successive approximation with $\varphi_{0}$ positive and nondecreasing on $[a, b]$, we have the representation

$$
\begin{aligned}
A^{n}\left(\varphi_{0}\right)(x) & =f(x)+\alpha \cdot(\text { Pos }) \int_{a}^{b} R_{n}(x, t ; \alpha) f(t) d P_{\lambda}(t) \\
& +\alpha^{n+1} \cdot(P o s) \int_{a}^{b} K_{n+1}(x, t) \varphi_{0}(t) d P_{\lambda}(t) \\
& =f(x)+\alpha \cdot \sup \left\{R_{n}(x, t ; \alpha) f(t) \lambda(t) ; t \in[a, b]\right\} \\
& +\alpha^{n+1} \cdot \sup \left\{K_{n+1}(x, t) \varphi_{0}(t) \lambda(t) ; t \in[a, b]\right\}
\end{aligned}
$$

where

$$
R_{n}(x, t ; \alpha)=\sum_{j=1}^{n} \alpha^{j-1} K_{j}(x, t) .
$$

Also, for the solution $\varphi^{*}(x)$ we have the representation

$$
\begin{gathered}
\varphi^{*}(x)=f(x)+\alpha \cdot(\text { Pos }) \int_{a}^{b} R(x, t ; \alpha) f(t) d P_{\lambda}(t) \\
=f(x)+\alpha \cdot \sup \{R(x, t ; \alpha) f(t) \lambda(t) ; t \in[a, b]\}, x \in[a, b],
\end{gathered}
$$

with

$$
R(x, t ; \alpha)=\sum_{j=1}^{\infty} \alpha^{j-1} \cdot K_{j}(x, t), x, t \in[a, b] .
$$

Proof. Taking $M=K(b, b)$, the hypothesis in Theorem 3.1 are fulfilled, fact which implies that there exists uniquely $\varphi^{*}$ satisfying (3.1).

It remains to deal with the sequence of successive approximations.

Let us choose $\varphi_{0}$ be positive and nondecreasing on $[a, b]$ (clearly it follows that $\varphi_{0}$ is bounded too). We get

$$
\begin{aligned}
\varphi_{1}(x)= & A\left(\varphi_{0}\right)(x)=f(x)+\alpha \cdot(\text { Pos }) \int_{a}^{b} K(x, s) \varphi_{0}(s) d P_{\lambda}(s) \\
& =f(x)+\alpha \cdot \sup \left\{K(x, s) \varphi_{0}(s) \lambda(s) ; s \in[a, b]\right\},
\end{aligned}
$$

which from the hypothesis immediately implies that $\varphi_{1}(x) \geq 0$, for all $x \in[a, b]$ and $\varphi_{1}$ is nondecreasing (and therefore bounded) on $[a, b]$.

Also, $\varphi_{1}$ is the sum of two positive and both nondecreasing functions on $[a, b]$. Since it is easy to prove that if $F$ and $G$ are both nondecreasing on $[a, b]$ then

$$
\begin{gathered}
(P o s) \int_{a}^{b}[F(s)+G(s)] d P_{\lambda}(s)=\sup \{(F(s)+G(s)) \lambda(s) ; s \in[a, b]\} \\
=F(b) \lambda(b))+G(b) \lambda(b)=\sup \{F(s) \lambda(s) ; s \in[a, b]\}+\sup \{G(s) \lambda(s) ; s \in[a, b]\}
\end{gathered}
$$


we obtain

$$
=(P o s) \int_{a}^{b} F(s) d P_{\lambda}(s)+(\operatorname{Pos}) \int_{a}^{b} G(s) d P_{\lambda}(s)
$$

$$
\begin{gathered}
\varphi_{2}(x)=f(x)+\alpha \cdot(\text { Pos }) \int_{a}^{b} K(x, t) \cdot \varphi_{1}(t) d P_{\lambda}(t) \\
=f(x)+\alpha \cdot(\text { Pos }) \int_{a}^{b} K(x, t) f(t) d P_{\lambda}(t) \\
+\alpha^{2} \cdot(\text { Pos }) \int_{a}^{b} K(x, t)\left[(\text { Pos }) \int_{a}^{b} K(t, s) \varphi_{0}(s) d P_{\lambda}(s)\right] d P_{\lambda}(t) .
\end{gathered}
$$

If, for each fixed $x \in[a, b]$ we denote $F(t, s)=K(x, t) \cdot K(t, s) \cdot \varphi_{0}(s)$, then $F(t, s) \geq 0$ for all $t, s \in[a, b]$ and $F(t, s)$ is nondecreasing in each variable $t$ and $s$. Also, since $F$ is bounded on $[a, b] \times[a, b]$, it follows that we can write

$$
\begin{aligned}
(\text { Pos }) \int_{a}^{b} K(x, t)\left[(\text { Pos }) \int_{a}^{b} K(t, s) \varphi_{0}(s) d P_{\lambda}(s)\right] d P_{\lambda}(t) \\
=\sup \left\{K(x, t) \lambda(t) \cdot \sup \left\{K(t, s) \varphi_{0}(s) \lambda(s) ; s \in[a, b]\right\} ; t \in[a, b]\right\} \\
=\sup \left\{\sup \{K(x, t) K(t, s) \lambda(t) ; t \in[a, b]\} \cdot \varphi_{0}(s) \lambda(s) ; s \in[a, b]\right\} \\
=\sup \left\{K_{2}(x, s) \varphi_{0}(s) \lambda(s) ; s \in[a, b]\right\} \\
=(\text { Pos }) \int_{a}^{b} K_{2}(x, s) \varphi_{0}(s) d P_{\lambda}(s),
\end{aligned}
$$

fact which leads to the formula

$\varphi_{2}(x)=f(x)+\alpha \cdot(P o s) \int_{a}^{b} K(x, t) \cdot f(t) d P_{\lambda}(t)+\alpha^{2} \cdot(P o s) \int_{a}^{b} K_{2}(x, s) \varphi_{0}(s) d P_{\lambda}(s)$.

Continuing these kinds of reasonings, step by step we easily get the recurrence formula

$$
\begin{gathered}
\varphi_{n+1}(x)=A^{n}\left(\varphi_{0}\right)(x) \\
=f(x)+\sum_{j=1}^{n} \alpha^{j} \cdot(\text { Pos }) \int_{a}^{b} K_{j}(x, t) f(t) d P_{\lambda}(t) \\
+\alpha^{n+1} \cdot(\text { Pos }) \int_{a}^{b} K_{n+1}(x, t) \varphi_{0}(t) d P_{\lambda}(t) \\
=f(x)+\alpha \cdot(\text { Pos }) \int_{a}^{b}\left[\sum_{j=1}^{n} \alpha^{j-1} K_{j}(x, t) f(t)\right] d P_{\lambda}(t) \\
+\alpha^{n+1} \cdot(\operatorname{Pos}) \int_{a}^{b} K_{n+1}(x, t) \varphi_{0}(t) d P_{\lambda}(t) \\
=f(x)+\alpha \cdot \sup \left\{R_{n}(x, t ; \alpha) f(t) \lambda(t) ; t \in[a, b]\right\} \\
+\alpha^{n+1} \cdot \sup \left\{K_{n+1}(x, t) \varphi_{0}(t) \lambda(t) ; t \in[a, b]\right\} .
\end{gathered}
$$

Now, by mathematical induction we easily can prove that

$$
0 \leq K_{n+1}(x, t) \leq[K(b, b)]^{n+1},
$$


for all $x, t \in[a, b]$ and $n=0,1, \ldots$, . This immediately implies (even uniformly with respect to $x \in[a, b])$

$$
\begin{gathered}
0 \leq \lim _{n \rightarrow \infty} \alpha^{n+1} \cdot(\text { Pos }) \int_{a}^{b} K_{n+1}(x, t) \varphi_{0}(t) d P_{\lambda}(t) \\
\leq \lim _{n \rightarrow \infty}\left([\alpha \cdot K(b, b)]^{n+1} \cdot(\text { Pos }) \int_{a}^{b} \varphi_{0}(t) d P_{\lambda}(t)\right) \\
\leq \lim _{n \rightarrow \infty}\left([\alpha \cdot K(b, b)]^{n+1} \cdot\left\|\varphi_{0}\right\|\right)=0
\end{gathered}
$$

and

$$
0 \leq \sum_{j=1}^{\infty} \alpha^{j-1} K_{j}(x, t) f(t) \lambda(t) \leq \sum_{j=1}^{\infty}(\alpha \cdot K(b, b))^{j-1} \cdot[K(b, b) f(b)] .
$$

Therefore, for each fixed $x \in[a, b]$,

$$
R_{n}(x, t ; \alpha)=\sum_{j=1}^{n} \alpha^{j-1} K_{j}(x, t) f(t) \lambda(t),
$$

converges (for $n \rightarrow \infty$ ) to $R(x, t ; \alpha) \cdot f(t) \lambda(t)$, uniformly with respect to $t \in[a, b]$.

Applying now the formula

$$
\begin{aligned}
& \mid \sup \{\left.R_{n}(x, t ; \alpha) f(t) \lambda(t) ; t \in[a, b]\right\}-\sup \{R(x, t ; \alpha) f(t) \lambda(t) ; t \in[a, b]\} \mid \\
& \leq \sup \left\{\left|R_{n}(x, t ; \alpha) f(t) \lambda(t)-R(x, t ; \alpha) f(t) \lambda(t)\right| ; t \in[a, b]\right\},
\end{aligned}
$$

we immediately arrive to formula (3.3).

Remark 3.4. It is clear that Corollary 3.3 remains valid if in its statement we replace everywhere the word "nondecreasing" with the word "nonincreasing" and $K(b, b)$ with $K(a, a)$.

\section{Volterra possibilistic integral equations}

It is known that in the classical case, the Volterra integral equation has solution for any value of the parameter $\alpha$. Unfortunately, in the case of Volterra possibilistic integral equation, this fact does not hold in general. However, for some appropriate choices of the possibility measure $P_{\lambda}$ in equation (1.2), it has unique solution for any value of the parameter $\alpha$.

Let us make the notation

$$
B_{+}[a, b]=\left\{f:[a, b] \rightarrow \mathbb{R}_{+} ; f \text { is bounded and positive on }[a, b]\right\},
$$

endowed with the uniform norm $\|\cdot\|$.

For our purpose and taking into account the definition of the possibilistic integral in Definition 2.1, in the Volterra possibilistic integral equation in (1.2) we will consider a family of possibility measures depending on a parameter $\tau>0$, for which (1.2) formally becomes the nonlinear functional equation

$$
\varphi(x)=f(x)+\alpha \cdot \sup \left\{K(x, s, \varphi(s)) \cdot \lambda_{\tau}(s) ; s \in[a, x]\right\}, x \in[a, b], \tau>0,
$$

where $\lambda_{\tau}, \tau>0$ is a family of possibility densities defined as in Definition 2.1, (ii). 
The main result is the following.

Theorem 4.1. Let $P_{\lambda_{\tau}}$ be the possibilistic measure induced by the possibilistic distribution $\lambda_{\tau}$ on $[a, b], \tau>0$.

Let us suppose that

$$
K \in B_{+}([a, b] \times[a, b] \times \mathbb{R}),
$$

where $B_{+}([a, b] \times[a, b] \times \mathbb{R})$ denotes the space of all positive and bounded functions $g:[a, b] \times[a, b] \times \mathbb{R} \rightarrow \mathbb{R}_{+}$, there exists $L>0$ such that

$$
|K(x, s, u)-K(x, s, v)| \leq L \cdot|u-v|, \text { for all } x, s \in[a, b], u, v \in \mathbb{R}_{+}
$$

and that

$$
\lim _{\tau \rightarrow+\infty} \sup \left\{\lambda_{\tau}(s) ; s \in[a, b]\right\}=0 .
$$

Then there exists $\tau_{0}>0$ such that for any $f \in B_{+}[a, b]$, any $\alpha>0$ and any $\tau>\tau_{0}$, the Volterra possibilistic integral equation (4.1) has a unique solution $\varphi_{\tau} \in B_{+}[a, b]$.

Proof. For any $\varphi \in B_{+}[a, b]$, let us denote

$$
T_{\tau}(\varphi)(x)=\sup \left\{K(x, s, \varphi(s)) \lambda_{\tau}(s) ; s \in[a, x]\right\}, x \in[a, b], \tau>0 .
$$

It is well-defined for any fixed arbitrary $x \in[a, b]$, because from hypothesis on $K$ and $\lambda_{\tau}(s)$, it easily follows that as function of $s, K(x, s, \varphi(s))$ there exists $M>0$ such that $|K(x, s, \varphi(s))| \lambda_{\tau}(s) \leq M$, for all $x, s \in[a, b]$. In what follows, we prove that $T_{\tau}(\varphi) \in B_{+}[a, b]$. For any fixed $x \in[a, b]$, we immediately get

$$
\begin{aligned}
T_{\tau}(\varphi)(x) & =\left|T_{\tau}(\varphi)(x)\right|=\left|\sup \left\{K(x, s, \varphi(s)) \lambda_{\tau}(s) ; s \in[a, x]\right\}\right| \\
& \leq \sup \left\{\left|K(x, s, \varphi(s)) \lambda_{\tau}(s)\right| ; s \in[a, x]\right\} \leq M .
\end{aligned}
$$

In conclusion $T_{\tau}(\varphi) \in B_{+}[a, b]$ and this also implies that $A_{\tau}(\varphi)=f+\alpha \cdot T_{\tau}(\varphi) \in$ $B_{+}[a, b]$.

Therefore, by using the hypothesis (4.3) and (4.4) too, we immediately obtain

$$
\begin{gathered}
\left|T_{\tau}(\varphi)(x)-T_{\tau}(\psi)(x)\right| \\
\leq \sup \left\{\left|K(x, s, \varphi(s)) \cdot \lambda_{\tau}(s)-K(x, s, \psi(s)) \cdot \lambda_{\tau}(s)\right| ; s \in[a, x]\right\} \\
\leq L \sup \{|\varphi(s)-\psi(s)| ; s \in[a, x]\}
\end{gathered}
$$

and

$$
\begin{gathered}
\left|A_{\tau}(\varphi)(x)-A_{\tau}(\psi)(x)\right|=\alpha \cdot\left|T_{\tau}(\varphi)(x)-T_{\tau}(\psi)(x)\right| \\
\leq \alpha \cdot L \sup \left\{|\varphi(s)-\psi(s)| \lambda_{\tau}(s) ; s \in[a, x]\right\} \\
\leq \alpha \cdot L \cdot \sup \left\{\lambda_{\tau}(s) ; s \in[a, b]\right\} \cdot\|\varphi-\psi\|,
\end{gathered}
$$

which immediately implies

$$
\begin{gathered}
d\left(A_{\tau}(\varphi), A_{\tau}(\psi)\right):=\left\|A_{\tau}(\varphi)-A_{\tau}(\psi)\right\| \\
\leq \alpha \cdot L \cdot \sup \left\{\lambda_{\tau}(s) ; s \in[a, b]\right\} \cdot\|\varphi-\psi\|_{\tau}=\alpha \cdot L \cdot \sup \left\{\lambda_{\tau}(s) ; s \in[a, b]\right\} \cdot d(\varphi, \psi) .
\end{gathered}
$$

From condition (4.4), there exists $\tau_{0}$ such that for all $\tau>\tau_{0}>0$ to get

$$
\alpha \cdot L \cdot \sup \left\{\lambda_{\tau}(s) ; s \in[a, b]\right\}<1,
$$

therefore $d$ is a contraction on the complete metric space $B_{+}[a, b]$ and applying the Banach's fixed point theorem we arrive at the desired conclusion. 
Remark 4.2. An important particular case is when $K(x, s, v):=K(x, s) \cdot v$. In this case, condition (4.2) becomes $K \in B_{+}([a, b] \times[a, b])$ and it immediately implies the condition (4.3), with $L=\sup \{K(x, s) ; x, s \in[a, b]\}$.

Remark 4.3. There are very many simple examples of families of possibilistic distributions satisfying condition (4.4) in Theorem 4.1, like, for example,

$$
\lambda_{\tau}(s)=e^{-\tau|s|+1}, \quad \lambda_{\tau}(s)=\frac{s^{2}}{\tau}, \quad \lambda_{\tau}(s)=\frac{|\sin (s)|}{\tau}, \quad \tau>0
$$

and so on.

\section{References}

[1] Bede, B., Coroianu, L., Gal, S.G., Approximation by Max-Product Type Operators, Springer, New York, 2016.

[2] De Cooman, G., Possibility theory. I. The measure and integral-theoretic groundwork, Internat. J. Gen. Systems, 25(1997), no. 4, 291-323.

[3] Coroianu, L., Gal, S.G., Opris, B., Trifa, S., Feller's scheme in approximation by nonlinear possibilistic integral operators, Numer. Funct. Anal. Optim, 38(2017), 327-343.

[4] Dubois, D., Prade, H., Possibility Theory, Plenum Press, New York, 1988.

[5] Gal, S.G., Fredholm-Choquet integral equations, J. Integral Equations Appl., 31(2019), no. 2, 183-194.

[6] Gal, S.G., Volterra-Choquet integral equations, J. Integral Equations Appl., 31(2019), no. 4, 495-504.

[7] Gal, S.G., A possibilistic approach of the max-product Bernstein kind operators, Results Math., 65(2014), 453-462.

[8] Gal, S.G., On the laws of large numbers in possibility theory, Ann. Acad. Rom. Sci. Ser. Math. Appl., 11(2019), no. 2, 274-284.

[9] Gal, S.G., Approximation by polynomial possibilistic integral operators, Ann. Acad. Rom. Sci. Ser. Math. Appl., 12(2020), no. 1-2, 132-141.

\section{Sorin G. Gal}

Department of Mathematics and Computer Science,

University of Oradea,

Universitatii 1, 410087, Oradea, Romania,

and

Academy of Romanian Scientists

Splaiul Independentei No. 54, Bucharest 050094, Romania

e-mail: galso@uoradea.ro, galsorin23@gmail.com

Ionut T. Iancu

Department of Mathematics and Computer Science,

University of Oradea,

Universitatii 1, 410087, Oradea, Romania

e-mail: ionutz.tudor.iancu@gmail.com 